\title{
Surface Recombination and Collection Efficiency in Perovskite Solar Cells from Impedance Analysis
}

Isaac Zarazua ${ }^{1}$, Guifang $\mathrm{Han}^{2}$, Pablo P. Boix ${ }^{2}$, Subodh Mhaisalkar ${ }^{2,3}$, Francisco Fabregat-Santiago $^{1}$, Ivan Mora-Seró ${ }^{1}$, Juan Bisquert ${ }^{1,4}$, Germà Garcia-Belmonte ${ }^{1, *}$

1 Institute of Advanced Materials (INAM), Universitat Jaume I, 12071 Castelló, Spain

2 Energy Research Institute@NTU (ERI@N), Nanyang Technological University, Research Techno Plaza, X-Frontier Block, Level 5, 50 Nanyang Drive, 637553, Singapore

3 School of Materials Science and Engineering, Nanyang Technological University, 50 Nanyang Avenue, 639798 Singapore

4 Department of Chemistry, Faculty of Science, King Abdulaziz University, Jeddah 21589, Saudi Arabia

Email: garciag@uji.es

\section{Abstract}

The large diffusion lengths recurrently measured in perovskite single crystals and films signals small bulk nonradiative recombination flux and locate the dominant carrier recombination processes at the outer interfaces. Surface recombination largely determines the photovoltaic performance, governing reductions in short circuit current and open circuit voltage. Quantification of recombination losses is necessary to reach full understanding of the solar cell operating principles. Complete impedance model is given which connects capacitive and resistive processes to the electronic kinetics at the interfaces. Carrier collection losses affecting the photocurrent have been determined to equal $1 \%$. Photovoltage loss is linked to the decrease in surface hole density, producing 0.3 V-reduction with respect to the ideal radiative limit. Our approach enables a comparison among different structures, morphologies and processing strategies of passivation and buffer layers.

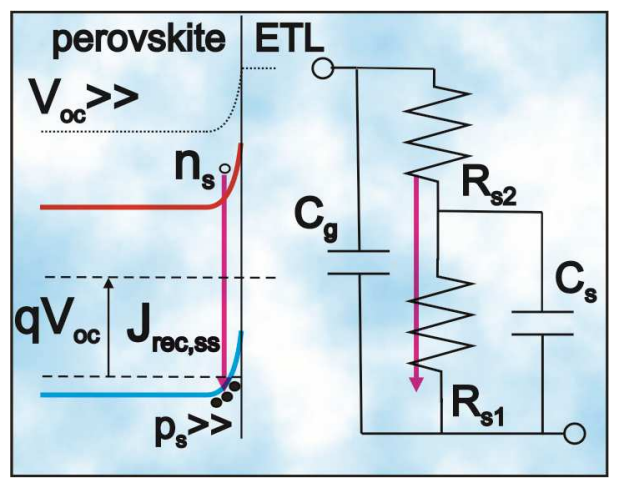

TOC figure 
Irruption of perovskite solar cell (PSC) technologies has shaken up applied and theoretical photovoltaics research. Power conversion efficiencies have reached in a few years values exceeding $22 \%$, propelling worldwide interest on perovskite materials and solar cell structures. ${ }^{1-2}$ Much is known about the physical properties of organolead halide perovskites. Carrier mobility and diffusivity are reported to be comparable to those of inorganic semiconductors ${ }^{3-5}$ so as to not severely limit charge collection efficiency at the outer contacts. In addition, several studies have related the superior performance of PSCs to the suppression of bulk nonradiative carrier recombination. ${ }^{6}$ The combination of these two observations, high carrier mobility and reduced internal recombination, lead to consider that the most important part of carrier recombination processes are more likely to occur at the interfaces between the perovskite absorber and extracting layers. ${ }^{7-9}$ According to this assumption, carrier losses by nonradiative recombination at the outer contacts play a crucial role determining the performance of perovskite solar cells, affecting both short circuit current and open circuit voltage. However, the quantification of the diminution in photovoltaic parameters in relation to interfacial electronic processes is still unsolved. Advances in this direction may contribute to a proper understanding of perovskite solar cell operational principles, enabling a direct comparison among the inclusion of different structures, morphologies, passivation strategies, buffer layers and selective contacts.

Impedance spectroscopy is a widely used technique that extracts dynamic operating parameters on complete solar cells. This experimental approach has become particularly fruitful in the application to a variety of different solar technologies. ${ }^{10-12}$ In essence, impedance spectroscopy captures the modulated (alternating) current in response to a small-amplitude modulated voltage stimulus. For data handling and description it is a common procedure to derive an equivalent circuit representing the internal resistive and capacitive response elements, which are connected to electronic/ionic mechanisms occurring at several, and hopefully differentiated, characteristic times. Despite its interpretative potential, the impedance response of perovskite solar cells is still not completely resolved. ${ }^{13-16}$ The analysis and mechanism identification of capacitive elements have raised certain attention in the last few years concluding that perovskite dielectric processes and interfacial charge accumulation dominate at the high- and lowfrequency response, respectively. ${ }^{17-18}$ However the detailed classification of the resistances seems to be particularly elusive. This is a central point for device understanding in so far as the dc behavior (and consequently the steady-state $J-V$ curve and efficiency) of the photovoltaic device is determined by the internal resistances. Several impedance studies point to the fact that outer interfaces somehow govern the overall resistive response of perovskite solar cells, ${ }^{19-20}$ in agreement to the excellent transport and reduced bulk recombination properties. But a detailed view is yet to be developed.

Here a selected combination of experimental conditions and perovskite solar cell structures reveals how carrier recombination processes occurring at the outer interfaces 
effectively dominate the operation of the measured solar cells. Particular and welldefined dependences of the resistive responses as a function of the light intensity and voltage allow for determination of the solar cell response time in relation to microscopic parameters. Moreover our approach permits the quantification of carrier collection losses affecting the photocurrent. It results in reductions of $\sim 1 \%$ for planar perovskite solar cells of regular and inverted architecture comprising different perovskite absorbers based on methylammonium ( $\mathrm{MAPbI}_{3}$ and $\mathrm{MAPbI}_{3-x} \mathrm{Cl}_{x}$ ) and formamidinium $\left(\mathrm{FAPbI}_{3-}\right.$ ${ }_{x} \mathrm{Cl}_{x}$ ), and contact materials as $\mathrm{TiO}_{2}$ and [6,6]-phenyl- $\mathrm{C}_{61}$-butyric acid methyl ester (PCBM). $\mathrm{MAPbI}_{3-x} \mathrm{Cl}_{x}$ and $\mathrm{FAPbI}_{3-x} \mathrm{Cl}_{x}$ have been prepared using $\mathrm{PbCl}$ precursor, presenting a low $\mathrm{Cl}$ content, however this nomenclature is used here as it is significantly extended in the literature.

Table 1 Photovoltaic parameters of the planar solar cells analyzed in this work measured in backward direction.

\begin{tabular}{|c|c|c|c|c|}
\hline $\begin{array}{l}\text { Thickness } \\
(\mathrm{nm})\end{array}$ & $\begin{array}{l}\text { Jsc } \\
\left.(\mathbf{m A ~ c m})^{-2}\right)\end{array}$ & $\begin{array}{l}\text { Voc } \\
(\mathrm{V})\end{array}$ & $\begin{array}{l}\text { FF } \\
(\%)\end{array}$ & $\begin{array}{l}\text { Efficiency } \\
(\%)\end{array}$ \\
\hline \multicolumn{5}{|l|}{$\begin{array}{l}\text { regular } \\
\mathrm{MAPbI}_{3} \\
\end{array}$} \\
\hline 471 & 16.77 & 1.101 & 66.26 & 12.23 \\
\hline 364 & 13.28 & 1.070 & 67.93 & 9.65 \\
\hline 300 & 12.30 & 1.044 & 70.11 & 9.00 \\
\hline \multicolumn{5}{|l|}{$\begin{array}{l}\text { Regular } \\
\text { FAPbI }_{3-x} \mathrm{Cl}_{x}\end{array}$} \\
\hline 300 & 19.36 & 0.960 & 53.77 & 9.99 \\
\hline 190 & 16.65 & 0.930 & 62.17 & 9.63 \\
\hline 130 & 14.36 & 0.870 & 62.48 & 7.81 \\
\hline \multicolumn{5}{|l|}{$\begin{array}{l}\text { Inverted } \\
\mathrm{MAPbI}_{3-x} \mathrm{Cl}_{x}\end{array}$} \\
\hline 400 & 17.62 & 0.939 & 54.40 & 9.01 \\
\hline 190 & 12.33 & 0.922 & 51.50 & 5.85 \\
\hline
\end{tabular}

Impedance spectroscopy response of regular structures. The impedance spectra of a set of different solar cells of regular $\left(\mathrm{FTO} / \mathrm{TiO}_{2} / \mathrm{MAPbI}_{3} /\right.$ spiro-OMeTAD/Au and $\mathrm{FTO} / \mathrm{TiO}_{2} / \mathrm{FAPbI}_{3-x} \mathrm{Cl}_{x} /$ spiro-OMeTAD/Au) planar structures of varying perovskite layer thickness have been checked in two different experimental conditions: either at short circuit (zero bias) or by applying a voltage equal to the open circuit voltage $\left(V=V_{\text {oc }}\right)$, for different illumination intensities ranging from 1 up to $100 \mathrm{~W} \mathrm{~cm}^{-2}$. Impedance spectra under illumination have been registered after leaving the solar cell to relax in the dark for at least $2 \mathrm{~min}$. This measuring procedure addresses either charge 
extraction (dominating the short circuit conditions) or charge recombination (determining the open circuit conditions) allowing for a full exploration the solar cell in different dynamic states. Photovoltaic parameters are listed in Table 1 for a close comparison and $J-V$ curves are included as Supporting Information (Figure S1 and S2) along with preparation procedures. The impedance response features two kinetically separated processes clearly exhibiting distinguishable arcs (i.e. with different time constants) for all the analyzed samples. Figure 1a shows an example of the impedance plots as a function of the irradiation intensity in which the resistive components of the two arcs shrink as the illumination increases. The equivalent circuit analysis used here relies upon previous findings that connect the impedance response to the properties of the solar cell dielectric bulk and outer interfaces. ${ }^{19-21}$ Apart from the external series resistance $R_{\mathrm{S}}$ accounting for the ohmic contribution of contacts and wires, the equivalent circuit comprises two capacitive $\left(C_{g}\right.$ and $\left.C_{\mathrm{S}}\right)$ and two resistive $\left(R_{2}\right.$ and $\left.R_{1}\right)$ elements, as drawn in Figure 1c. The capacitive elements have been explicitly labeled according to the established interpretation, associated to the dielectric response of the perovskite layer $C_{g}$, and to the surface charge accumulation at the interfaces $C_{\mathrm{S}}$. Whereas $C_{g}$ dominates the capacitive response in the high-frequency part $(>1 \mathrm{kHz})$ of the spectra (Figure 1b), $C_{\mathrm{s}}$ responds to the prominent low-frequency $(\sim 1 \mathrm{~Hz})$ mechanisms that govern the solar cell operation (Figure 1b). These two capacitances have been extensively analyzed in previous works. ${ }^{17,22}$ The dielectric constant of the light absorbing perovskite layer $\varepsilon$ gives rise to the geometrical capacitance as $C_{g}=\varepsilon \varepsilon_{0} / d$, being $d$ the layer thickness and $\varepsilon_{0}$ the permittivity of the free space. $C_{\mathrm{s}}$ appears as a consequence of interface accumulation processes, either of ionic character in the dark, or electronic under illumination. ${ }^{18}$ Although several studies have discussed the origin of the different electrical features of the perovskite solar cells, the lack of a complete model, including resistive elements, prevents a full understanding of how these circuit elements define the performance of the cells. Here we aim at progressing in the understanding of the resistive mechanisms that ultimately establish the steady-state response of the $J-V$ curves.
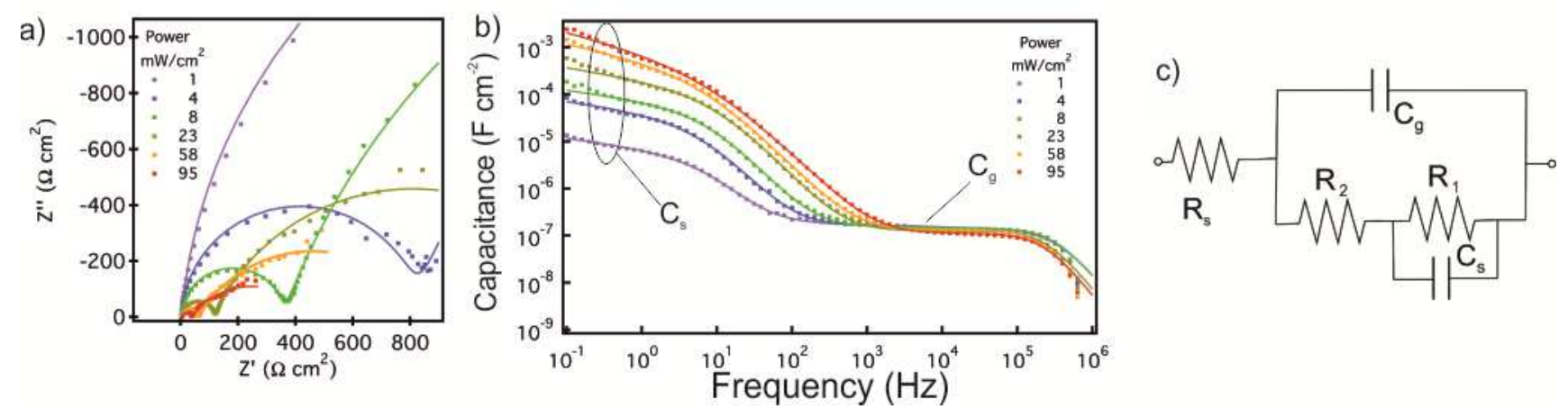

Figure 1. a) Example of impedance plot $\left(Z^{\prime \prime}-Z^{\prime}\right)$ measured in short circuit conditions at different irradiation intensities corresponding to perovskite solar cells of planar structure $\mathrm{FTO} / \mathrm{TiO}_{2} / \mathrm{MAPbI}_{3} /$ spiro-OMeTAD/Au. b) Example of capacitance spectra corresponding to the 
conditions in a). Solid lines correspond to fits using the equivalent circuit c). $R_{\mathrm{S}}$ accounts for the series resistance. $C_{g}$ and $C_{\mathrm{S}}$ are the geometrical capacitance and surface accumulation capacitance, respectively. $R_{2}$ and $R_{1}$ are related to the surface recombination current flux.

An example of the fitting results of a set of $\mathrm{MAPbI}_{3}$-based cells of varying absorber layer thickness is presented in Figure 2. A similar analysis for the case of $\mathrm{FAPbI}_{3-x} \mathrm{Cl}_{x^{-}}$ based cells is given in Figure S4. In short circuit conditions (Figure 2a and 2b), $C_{g}$ always results in values that are independent on the irradiation intensity and on the order of $C_{g} \approx 0.1 \mu \mathrm{F} \mathrm{cm} \mathrm{cm}^{-2}$ with larger values for thinner perovskite layers in agreement with its dielectric character. ${ }^{17}$ On the contrary, $C_{\mathrm{s}}$ rises by orders of magnitude proportionally to the light intensity, as noted in the plot, reaching values as high as $C_{\mathrm{S}} \approx 1 \mathrm{mF} \mathrm{cm}^{-2}$ at 1 sun illumination as previously reported. ${ }^{22}$ Interestingly, the resistive components follow the inverse trend with light intensity, with the low-frequency resistance $R_{1}$ showing larger values than the high-frequency contribution $R_{2}$. The fact that resistive elements and accumulation capacitance behave in the opposite way (strictly proportional) with irradiation intensity leads us to consider that both stem from a common mechanism as discussed later on.

Figure $2 \mathrm{c}$ and $2 \mathrm{~d}$ show the parameter variation of the $\mathrm{MAPbI}_{3}$-based cells at open circuit (see Figure S3 for $J-V$ curves). Here values are displayed as a function of the reached $V_{\mathrm{Oc}}$ at each illumination. The same general trends are again repeated: an essentially constant $C_{g}$ and an increasing $C_{\mathrm{S}}$ with higher illumination intensity. Recently, $C_{\mathrm{S}}$ has been interpreted in terms of the light-induced built-up of an electronic accumulation zone formed by majority carrier (holes) in the vicinity of the cathode contact $\left(\mathrm{TiO}_{2} /\right.$ perovskite interface). ${ }^{18}$ The model in Figure 3 accounts for the cathode contact in open circuit conditions. The accumulation layer corresponds to an upward band bending zone with extension equaling the Debye length of the background hole density $p_{0}$ as $L_{D}=\sqrt{\varepsilon \varepsilon_{0}} k_{\mathrm{B}} T / q^{2} p_{0}$. Here $q$ accounts for the elementary charge and $k_{\mathrm{B}} T$ is the thermal energy. For typical background of majorities $p_{0} \sim 10^{17} \mathrm{~cm}^{-3}$, which corresponds to the doping density, $L_{D} \sim 10 \mathrm{~nm}$. This model relates $C_{\mathrm{s}}$ to the voltage derivative of the surface charge $p_{\mathrm{S}}=p_{0} L_{D} \sqrt{2} e^{q V / 2 k_{\mathrm{B}} T}$ as $C_{\mathrm{S}}=q d p_{\mathrm{S}} / d V$, and predicts a variation of $C_{\mathrm{S}}$ with the voltage following the expression

$$
C_{\mathrm{s}}=\frac{\varepsilon \varepsilon_{0}}{\sqrt{2} L_{D}} \exp \left(\frac{q V}{2 k_{\mathrm{B}} T}\right) .
$$

In this model part of the $V_{\mathrm{oc}}$ is built-up at the interface as a consequence of the formation of a hole accumulation zone $V_{\mathrm{oc}}^{\mathrm{acc}}$, represented in Figure 3 as an offset in the vacuum level. The rest of the photovoltage $\left(V_{\mathrm{oc}} \approx 0.4-0.5 \mathrm{~V}\right)$ corresponds to the suppression of the downward band bending achieved at very low light intensities. ${ }^{18}$ Thus the surface charge accumulation $p_{\mathrm{s}}$ attains pronounced values for high light intensity (Figure $3 b$ and $c$ ). It is in turn related to a dielectric capacitor (Equation 1), which varies exponentially with voltage with slope $1 / 2 k_{\mathrm{B}} T$. This response was verified in the case of $\mathrm{FAPbI}_{3-x} \mathrm{Cl}_{x}$-based cells (Figure S4c), ${ }^{18}$ and it is now corroborated for $\mathrm{MAPbI}_{3}$ (Figure 
2c). The voltage-dependence of Equation 1, along with the huge values attained by the capacitances, reinforces the interfacial character of the underlying mechanism. The resistive response (Figure 2d) exhibits the opposite variation with $V_{\text {oc }}$, presenting an inverse slope $-1 / 2 k_{\mathrm{B}} T$. Again, both resistive elements appear to be somehow connected to the accumulation capacitor by a common mechanism. It is also noticeable that open circuit resistances (Figure 2d) are always smaller than their short circuit counterparts. This fact is explained by the inverse relation between resistances and recombination current as later explained. At open circuit recombination current completely cancels photogeneration current thereby reducing resistances. The most significant reduction occurs for the low-frequency resistance, $R_{1}$, that presents values within the range $10^{5}-10^{3} \Omega \mathrm{cm}^{2}$ at short circuit, but diminishes to $10^{3}-10^{1} \Omega \mathrm{cm}^{2}$ at open circuit.

In order to further investigate the solar cell operating mechanism on the basis of the impedance analysis, a standard procedure consists of calculating the time constants corresponding to the equivalent circuit in Figure 1c. In principle one can define two separated times as the products $\tau_{2}=R_{2} C_{g}$ and $\tau_{1}=R_{1} C_{\mathrm{S}}$. These time constants are associated with the high- and low-frequency arcs, provided that $C_{\mathrm{s}} \gg C_{g}$ as it is the case. Figure 4 shows the two time constants calculated for regular planar structures with $\mathrm{MAPbI}_{3}$ (Figure $4 \mathrm{a}$ and $4 \mathrm{~b}$ ) and $\mathrm{FAPbI}_{3-x} \mathrm{Cl}_{x}$ (Figure $4 \mathrm{c}$ and $4 \mathrm{~d}$ ). The shorter time constant $\tau_{2}$ follows the trend marked by $R_{2}$. Since $C_{g}$ is decoupled from the resistive element $R_{2}$, the corresponding time constant cannot be regarded as a characteristic time of any physical process. In contrast, the longer time constant $\tau_{1}$ exhibits lightindependent values both in short and open circuit conditions, and it always lies in the range $0.1-1 \mathrm{~s}$. The light-independent behavior and similar order of magnitude of these time constants are strong indications of the coupling between $R_{1}$ and $C_{\mathrm{S}}$ in a common kinetic mechanism. It is worth noting that this last observation confirms the opposite variation with light intensity shown in Figure 2 and S4. We next proceed to derive a model accounting for the mechanism behind the low-frequency impedance response, which is linked to the surface recombination processes. 

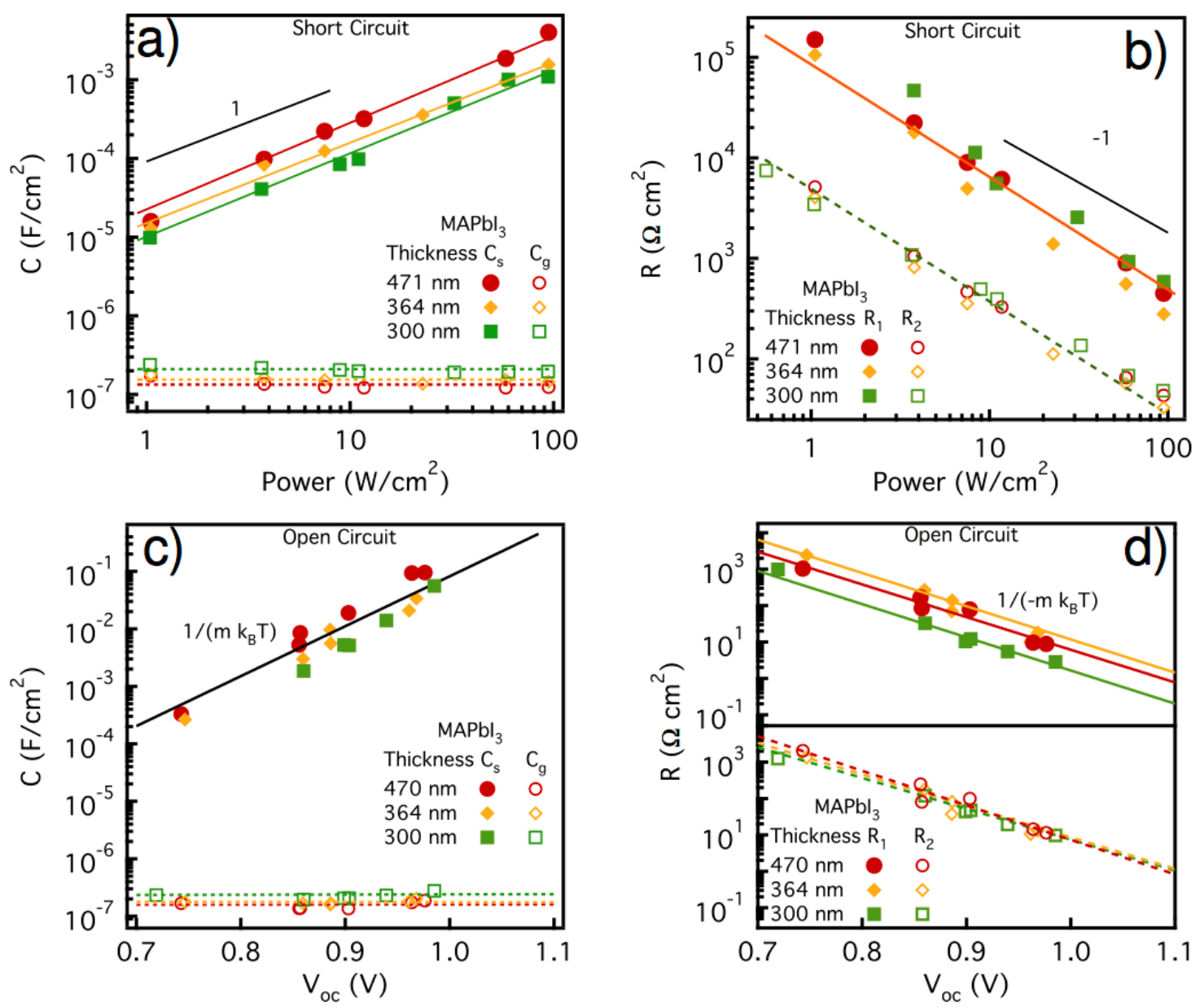

Figure 2. a) Capacitances and b) resistances at short circuit conditions for varying illumination intensities of $\mathrm{MAPbI}_{3}$-based planar solar cells of regular structure with different layer thickness as indicated. Proportionality lines between capacitances $(+1)$ and resistances $(-1)$ are indicated. c) Capacitances and d) resistances at open circuit conditions. Solid lines (low-frequency arc) and dashed lines (high-frequency arc) correspond to linear fits with $m$ approaching 2. In c) $m=1.90 \pm 0.17$ and d) $m=1.94 \pm 0.08$.
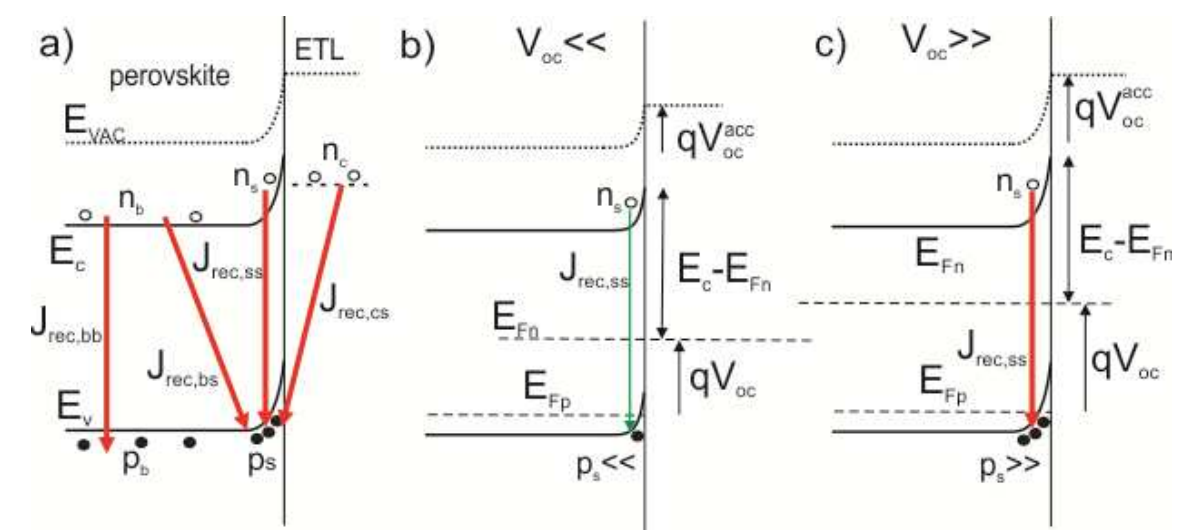

Figure 3. a) Energy band diagram of the accumulation zone at the perovskite near the 
cathode contact (perovskite/electron transport layer) in open circuit conditions. The schematic signals possible different electron-hole recombination routes. It is distinguished among purely bulk recombination flux $J_{\text {rec, bb }}$ (carriers located at the perovskite bulk), purely surface recombination $J_{\text {rec,ss }}$ (carriers located at the interfacial accumulation zone), mixed bulksurface recombination $J_{\text {rec,bs }}$ (perovskite bulk electrons and interfacial holes), and mixed cathode-surface recombination $J_{\text {rec,cs }}$ (cathode material electrons and interfacial holes). The shown downward fluxes schematically account for the recombination routes without specifying mechanistic details. Band diagram shows the formation on a majority carrier (hole) accumulation zone in the vicinity of the cathode contact in open circuit conditions. Two cases are shown here: $\mathrm{b}$ ) the accumulation zone produces a slight upward band bending for $p_{\mathrm{s}}<<$ (slight illumination intensity producing reduced $V_{\mathrm{oc}}$ ) or c) larger band bending for $p_{\mathrm{s}} \gg>$ (high illumination intensity with $V_{\mathrm{Oc}}>>$ ). In this model part of $V_{\mathrm{oc}}$ is built-up at the interface as a consequence of the formation of a hole accumulation zone $V_{\mathrm{oc}}^{\mathrm{acc}}$, represented here as an offset in the vacuum level. The rest of the photovoltage $\left(V_{\mathrm{Oc}} \approx 0.4-0.5 \mathrm{~V}\right)$ corresponds to the suppression of the downward band bending achieved at very low light intensities. ${ }^{18}$ The electron density at the interface $n_{\mathrm{s}}$ is illumination-independent as the local distance between $E_{F n}$ and the perovskite conduction band $E_{\mathrm{c}}$ is constant.
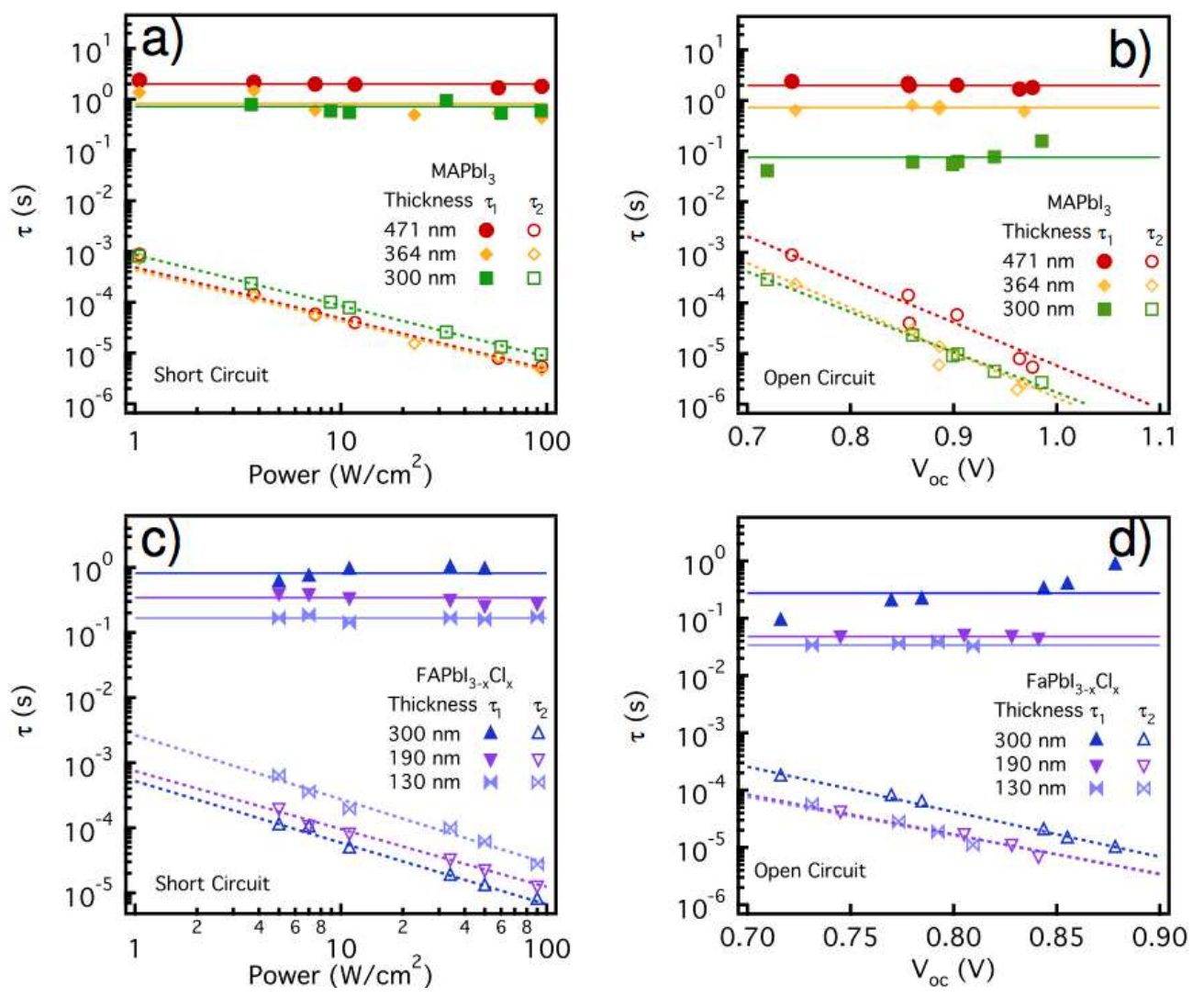

Figure 4. Response time calculated from the $R C$ product for the low frequency arc (solid line) and high-frequency arc (dashed line), respectively. a) short circuit and b) open circuit for $\mathrm{MAPbI}_{3}$-based planar solar cells, and c) short circuit and d) open circuit for $\mathrm{FAPbI}_{3-x} \mathrm{Cl}_{x}$-based 
planar solar cells.

Surface recombination. The large diffusion lengths recurrently measured in perovskite single crystals and films are often interpreted as an effect of a low bulk nonradiative recombination rate, which let us assume that the dominant recombination processes of perovskite solar cells are located at the outer interfaces. Moreover, the large hole concentration resulting from the formation of interfacial accumulation zones can promote recombination events in the vicinity of the contact. This view connects the resistive impedance response to the partial current reduction (at short circuit) or its complete suppression (at open circuit). Interestingly, the two resistances employed in the model exhibit extremely similar behavior as a function of light-intensity (Figure 2b) and photovoltage (Figure 2d). This fact suggests a common origin for both resistances, which are connected in series in the equivalent circuit of Figure 1c. However, they are kinetically linked to different capacitive processes giving rise to well-differentiated time constants, $\tau_{2}=R_{2} C_{g}$ and $\tau_{1}=R_{1} C_{\mathrm{S}}$. As discussed above several possibilities can be devised accounting for the reported similar light and voltage dependences of the resistances that ultimately appear in series. On the one hand it is likely that the overall recombination process is a trap-mediated, multistep event of a Schottky-Read-Hall kind. ${ }^{23}$ This microscopic recombination mechanism is preceded by the capture of an electron and a hole by a surface recombination center where the recombination event finally takes place. Two resistances are expected for the separate carrier capture processes of electrons and holes. Another possibility is the interpretation of $R_{1}$ as a purely transport mechanism preceding the final recombination event, which could well be coupled to the reported ionic movement. ${ }^{24} R_{1}$ might also contain information on recombination mechanisms mediated by bulk electrons. A deeper study of the charge dynamics could help to elucidate the specific origin of the components. Nevertheless, the underlying mechanism is certainly elusive, and it can generate complex kinetic responses including even inductive loops. ${ }^{20,25}$

By examining Figure 3a one can observe different recombination routes emphasizing the distinction between purely bulk- from interface-mediated recombination events. From previous comments, electron-hole recombination $J_{\text {rec,bb }}$ at the perovskite bulk is discarded as a dominant process of the observed resistance. Therefore we will focus in this paper on the surface recombination fluxes, while recognizing that the situation may be different in extremely high efficiency devices with substantial external radiative quantum efficiency, where many surface recombination losses would need to be suppressed. A charge transfer process between electrons in $\mathrm{TiO}_{2}$ and accumulated holes in the interface $J_{\text {rec,cs }}$ (Fig. 3a) does not obey the found carrier statistics, what it leads us to disregard this route in our model. The distinction between purely surface recombination $J_{\text {rec,ss }}$ from $J_{\text {rec,bs }}$, which involves electrons located at the perovskite bulk, is certainly difficult in experimental terms. Here the total surface recombination current $J_{\text {rec,s }}$ is written as a function of the surface density of majority carriers $p_{\mathrm{s}}$ (per 
unit area) and the minority (electron) density $n$ (per unit volume), either at the perovskite layer bulk $n_{\mathrm{b}}$ or the collecting interface $n_{\mathrm{s}} \cdot{ }^{26}$ Two terms are then considered that correspond to the recombination fluxes involving accumulated surface holes, as $J_{\text {rec }, \mathrm{s}}=J_{\text {rec }, \mathrm{ss}}+J_{\text {rec,bs }}$ (see Figure 3)

$$
J_{\mathrm{rec}, \mathrm{s}}=q k_{\mathrm{s}} p_{\mathrm{s}} n_{\mathrm{s}}+q k_{\mathrm{b}} p_{\mathrm{s}} n_{\mathrm{b}}
$$

where $k_{\mathrm{s}}$ and $k_{\mathrm{b}}$ are recombination coefficients of the purely surface and mediated through bulk electron loss mechanisms (with dimensions $\mathrm{cm}^{3} \mathrm{~s}^{-1}$ ). Equation 2 is a general expression that does not specify the microscopic process by which recombination occurs. It is valid for a band-to-band interaction as well as for a trapassisted mechanism, in which case bulk $n_{\mathrm{b}}$ and interface $n_{\mathrm{s}}$ contain information about trap carrier densities. The equation assumes a recombination process of bimolecular origin - similar to the one encountered in organic solar cells - and it specifically highlights that recombination events mainly occur at the perovskite/contact interface where $p_{\mathrm{s}}$ attains significantly large values. Insights on the specific processes dominating the recombination would permit a precise formulation of the recombination coefficients, which is unknown at the moment.

Irrespective to the microscopic details of the physical process giving rise to carrier recombination, our findings entail that the total surface recombination resistance should result from the series connection of a multistep process as $R_{\text {rec, } \mathrm{s}}=R_{1}+R_{2}$. At short circuit conditions the addition of $R_{2}$ is certainly a minor contribution to the effective $R_{\text {rec,s }}$. However, at open circuit conditions the inclusion of $R_{2}$ with $R_{1} \approx R_{2}$ practically doubles the value of $R_{\text {rec,s }}$. The invariable time constant reinforces the identification of $R_{1}+R_{2}$ as the overall recombination resistance. As occurs for the recombination resistance in other kind of solar cells, ${ }^{27} R_{\text {rec,s }}$ can be obtained from the voltage derivative of the overall recombination current $J_{\text {rec,s }}$, which in this case takes place at the outer interface.

$$
R_{\mathrm{rec}, \mathrm{s}}=\left(\frac{d J_{\mathrm{rec}, \mathrm{s}}}{d V}\right)^{-1}
$$

Combining Equation 2 and 3 the surface recombination resistance results in

$$
R_{\mathrm{rec}, \mathrm{s}}^{-1}=q k_{\mathrm{s}} n_{\mathrm{s}} \frac{d p_{\mathrm{s}}}{d V}+q k_{\mathrm{b}} n_{\mathrm{b}} \frac{d p_{\mathrm{s}}}{d V}+q k_{\mathrm{b}} p_{\mathrm{s}} \frac{d n_{\mathrm{b}}}{d V}
$$

assuming that $n_{\mathrm{s}}$ is a voltage-independent parameter, as next explained. Also $k_{\mathrm{s}}$ and $k_{\mathrm{b}}$ are regarded as constant rates. It is worth to remark that $C_{\mathrm{s}}=q d p_{\mathrm{s}} / d V$ corresponds to the accumulation capacitance (per unit surface) previously introduced in Equation 1, and $c_{\mu}=q d n_{\mathrm{b}} / d V$ accounts for the chemical capacitance of bulk electrons (per unit volume). Equation 4 can be expressed as

$$
R_{\mathrm{rec}, \mathrm{s}}^{-1}=k_{\mathrm{s}} n_{\mathrm{s}} C_{\mathrm{s}}+k_{\mathrm{b}} n_{\mathrm{b}} C_{\mathrm{s}}+k_{\mathrm{b}} p_{\mathrm{s}} c_{\mu}
$$

As observed, the third summand is proportional to $c_{\mu}$. Recent calculations have resulted 
in extremely low values for $c_{\mu}$ for $\mathrm{MAPbI}_{3}$, even below the perovskite layer geometrical capacitance $C_{g}$ that completely masks it. ${ }^{28}$ This permits to rule out its contribution in comparison to the other two terms in Equation 5. As a consequence, Equation 5 enables the interpretation of the time constant featuring the surface recombination process as

$$
\tau_{\mathrm{s}}=R_{\mathrm{rec}, \mathrm{s}} C_{\mathrm{s}}=\frac{1}{k_{\mathrm{s}} n_{s}+k_{\mathrm{b}} n_{\mathrm{b}}} .
$$

From Equation 6 the recombination time constant contains two terms determined by surface and bulk electron densities. Strictly speaking, $\tau_{\mathrm{s}}$ cannot be properly considered as a carrier lifetime because the $C_{\mathrm{s}}$ nature is dielectric and not chemical. Recalling the voltage-independent characteristic times of Figure 4, it is inferred that the term $k_{\mathrm{b}} n_{\mathrm{b}}$ should have only a minor influence on $\tau_{\mathrm{s}}$ because $n_{\mathrm{b}}$ is expected to increase with voltage (light intensity) producing, as a consequence, a reduction in $\tau_{\mathrm{s}}$ that is not observed at all. This last argument simplifies Equation 6 in such a way that

$$
\tau_{\mathrm{s}}=\frac{1}{k_{\mathrm{s}} n_{s}}
$$

indicating that $\tau_{\mathrm{s}}$ is determined by electrons at the interface $n_{s}$. It is worth recalling that the product $k_{\mathrm{s}} p_{\mathrm{s}}$ has the dimension of a velocity and is usually assimilated to surface recombination velocity. We next elaborate a rationale on the constant value that $n_{s}$ should exhibit for the sake of consistency with voltage-independent characteristic times of Figure 4.

A diagram of the surface recombination process in open circuit conditions is drawn in Figures $3 \mathrm{~b}$ and $\mathrm{c}$. It is observed how bands bend upward near the contact because of the space-charge layer formed by holes (accumulation zone). It is precisely that space charge region that sustains part of the photovoltage. The electron Fermi level offset with respect to the perovskite conduction band changes upon illumination at the material bulk as schematically drawn in Figures $3 \mathrm{~b}$ and $\mathrm{c}$, but $n_{\mathrm{s}}$ is illumination-independent at the interface because the local distance between $E_{F n}$ and the conduction band $E_{c}$ does not vary with light. In contrast to this, the hole surface density $p_{\mathrm{S}}$ grows as light intensity is increased. Thus, $n_{\mathrm{s}}$ is assumed as constant in the derivation of Equation 4 and 7 for a given cell configuration and measuring conditions. Also the constancy of $n_{\mathrm{s}}$ would mean saturation of trap states with electrons. It should be noted that Equation 7 will only be valid for illumination intensities which assure the formation accumulation zone. It is known from previous analysis ${ }^{18}$ that it generally occurs for $V_{\mathrm{oc}} \geq 0.4-0.5 \mathrm{~V}$ above which the photovoltage is held by the electrostatic potential built-up at the interface.

Recalling that $R_{\text {rec, } \mathrm{s}}=R_{1}+R_{2}$ as stated previously, one arrives to

$$
\tau_{\mathrm{s}}=\left(R_{1}+R_{2}\right) C_{\mathrm{S}}
$$

that corresponds to the experimental determination of Equation 7 considered as a measurement of the kinetics of surface recombination. A closer look at the $\tau_{\mathrm{s}}$ variation with absorber thickness and material reveals common trends. As it can be observed in 
Figure $5, \tau_{\mathrm{s}}$ always exhibits larger values at short circuit compared to open circuit conditions. This can be easily explained by the higher $n_{\mathrm{S}}$ attained at open circuit. Besides, $\tau_{\mathrm{s}}$ increases with thickness, which might indicate the reduction of $n_{\mathrm{s}}$ in the recombination zone for thicker absorbers. However, we cannot rule out here that the recombination microscopic properties of the contact (represented by $k_{\mathrm{S}}$ ) change with device processing and the chemistry of the interface. An estimation of $k_{\mathrm{S}}$ can be given by assuming electron density of order $n_{\mathrm{s}} \approx 10^{15} \mathrm{~cm}^{-3}$. From $\tau_{\mathrm{s}} \approx 0.1-1 \mathrm{~s}$, it is obtained $k_{\mathrm{S}} \approx 10^{-15}-10^{-16} \mathrm{~cm}^{3} \mathrm{~s}^{-1}$ for regular cells.

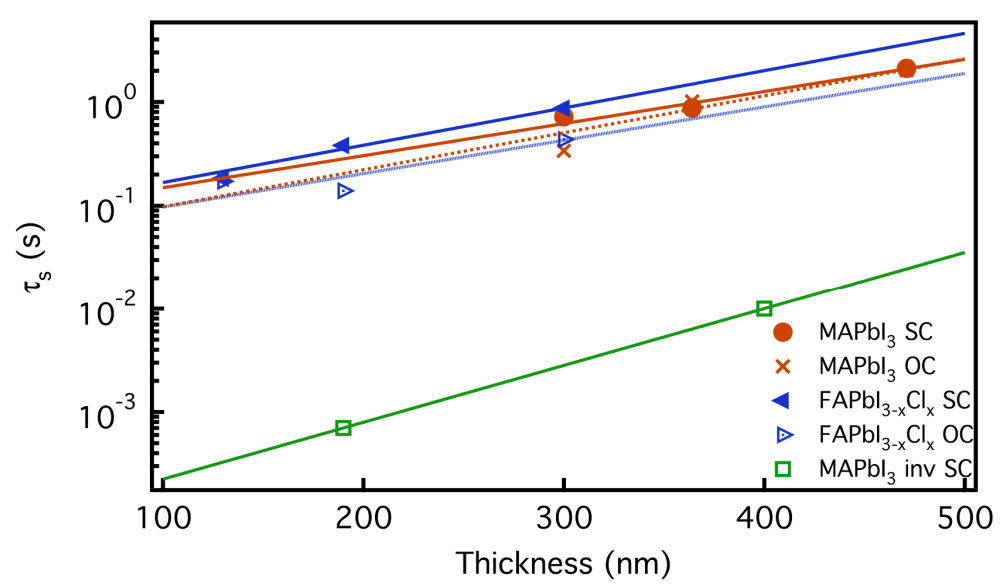

Figure 5. Surface recombination time calculated from impedance analysis as $\tau_{\mathrm{S}}=\left(R_{1}+R_{2}\right) C_{\mathrm{S}}$ exhibited by different perovskite solar cell structures in open circuit and short circuit conditions.

Response of inverted structures. In order to verify the generality of the experimental trends observed in Figure 2 for regular cells, we analyzed inverted perovskite solar cells comprising bottom organic hole transporting layers and fullerene-derivative top contacts with the structure ITO/PEDOT:PSS/MAPbI ${ }_{3-x} \mathrm{Cl}_{x} / \mathrm{PCBM} / \mathrm{Ag}$. Photovoltaic parameters are listed in Table 1; experimental details and $J-V$ curves (Figure S5) as Supporting Information. Inverted solar cells are known to exhibit reduced hysteresis $J-V$ curves. ${ }^{29}$ This effect has been recently connected to the reduced capacitance contribution these kind of solar cells present in the low-frequency part of the spectra. ${ }^{30}$ The general trends of the regular cell impedance response (Figure 1 and 2) are also observed in the case of inverted structures (Figure 6). Figure 6a shows the increase of the low-frequency capacitance with the light intensity, but exhibiting much lower values than those reported for $C_{\mathrm{s}}$ of regular structures. The high-frequency capacitance behavior is in good agreement to bulk dielectric properties, as expected for $C_{g}$. Resistance variation with light intensity (Figure 6b) also mimics the one observed in the previous analysis of regular structures. Hence, general response trends are reproduced with inverted solar cells, being the order of magnitude of $C_{\mathrm{S}}$ the only singular difference. This last observation indicates that the dissimilar $C_{\mathrm{S}}$ in the dark establishes the value onto which 
the light-induced excess accumulation capacitance is built up.

The similar pattern exhibited by regular and inverted structures leads us to consider the outlined surface recombination model as a valid approach of the operating modes of inverted cells as well. Recombination characteristic times exhibit light-independent values (Figure $6 \mathrm{c}$ ) although orders of magnitude $\left(\tau_{\mathrm{s}} \approx 10^{-3}-10^{-2} \mathrm{~s}\right)$ below those exhibited by regular cells (see Figure 5 for a close comparison). Assuming similar electron density in the vicinity of the contact one can readily calculate the surface recombination coefficient, which results within $k_{\mathrm{s}} \approx 10^{-12}-10^{-13} \mathrm{~cm}^{3} \mathrm{~s}^{-1}$ for inverted cells. This implies faster kinetics of recombination for fullerene contacts when compared to inorganic extracting layers. However we remark that surface recombination currents do not significantly differ among different structures as inferred by examining the resistance values of Figures 2 and 6 (see also Figure S6 for a close comparison). We next progress on that issue.
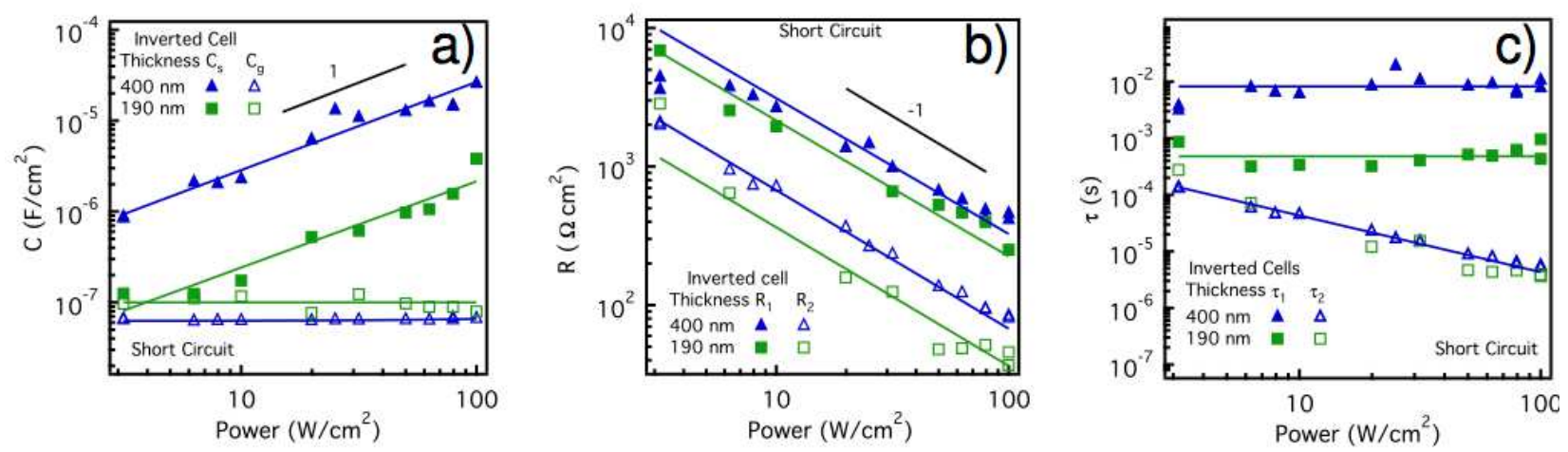

Figure 6. a) Capacitances and b) resistances at short circuit conditions for varying illumination intensities of $\mathrm{MAPbI}_{3-x} \mathrm{Cl}_{x}$-based planar solar cells of inverted structure with different layer thickness as indicated. c) Response time calculated from the $R C$ product for the low frequency arc (horizontal line) and high-frequency arc (inclined line), respectively

Determination of collection efficiency. The voltage modulation during impedance experiment changes the carrier (hole) local surface density as $\Delta p_{\mathrm{s}} / \Delta V=q p_{\mathrm{s}} / 2 k_{\mathrm{B}} T$. As a consequence of the exponential dependence of the local carrier density with voltage $p_{\mathrm{S}} \propto \exp \left(q V / 2 k_{\mathrm{B}} T\right)$, one readily arrives at the inversely proportional dependence of $R_{\text {rec,s }}$ on $J_{\text {rec,s }}$ from Equation 3

$$
J_{\text {rec }, \mathrm{s}}=\frac{2 k_{\mathrm{B}} T / q}{R_{\mathrm{rec}, \mathrm{s}}}
$$

At open circuit conditions, the photogeneration current $J_{\mathrm{ph}}$ is totally suppressed by recombination current $J_{\mathrm{ph}}=J_{\mathrm{rec}, \mathrm{s}(\mathrm{oc})}$. If we assume that photogeneration is a voltageindependent process, Equation 9 can be used to estimate $J_{\mathrm{ph}}$ and compare it with the actually measured $J_{\mathrm{sc}}$. At short circuit conditions part of the photogenerated carriers recombine at the outer interface while the main part of the carriers are drained off. This 
implies that the measured short circuit current is reduced by a term related to the carrier loss by surface recombination

$$
J_{\mathrm{sc}}=J_{\mathrm{ph}}-J_{\mathrm{rec}, \mathrm{s}(\mathrm{sc})}
$$

The ratio between recombination and photogenerated current allows defining the effective collection loss of the solar cell as

$$
\alpha=\frac{J_{\text {rec }, \mathrm{s}(\mathrm{sc})}}{J_{\mathrm{ph}}}=\frac{J_{\text {rec }, \mathrm{s}(\mathrm{sc})}}{J_{\text {rec }, \mathrm{s}(\mathrm{oc})}}=\frac{R_{\mathrm{rec}, \mathrm{s}(\mathrm{oc})}}{R_{\mathrm{rec}, \mathrm{s}(\mathrm{sc})}}
$$

which is supposed to be as small as possible for good extracting contacts. Equation 11 assumes that Equation 9 can be consistently applied also at short circuit conditions. This is obviously not justified here, since a physical model for the short circuit surface capacitances has yet to be addressed. In any case, for a solar cell mainly limited by surface recombination mechanisms, one can infer from Equation 11 that the collection efficiency is simply related to the ratio between recombination resistances measured at open circuit and short circuit. As can be seen in Table 2 collection losses calculated at 1 sun illumination intensity are always approximately of $1 \%$ irrespective of the contacts and architectures used. From Equation 10, one can infer $J_{\mathrm{sc}}=(1-\alpha) J_{\mathrm{ph}}$, being $\eta=1-\alpha$ the collection efficiency. The collection efficiency can be either calculated from $\eta=J_{\mathrm{sc}} / J_{\mathrm{rec}, \mathrm{s}(\mathrm{oc})}$ or $\eta=1-R_{\mathrm{rec}, \mathrm{s}(\mathrm{oc})} / R_{\mathrm{rec}, \mathrm{s}(\mathrm{sc})}$, and the comparison between these two procedures are used here as a test for consistency. This comparison is outlined in Table 1 validating the consistency of the interface recombination model. The main message of these data is about the slight difference between internal and external quantum efficiencies in this kind of solar technology.

Table 2. $\alpha$ collection loss in short circuit conditions, and collection efficiency calculated either from $\eta=J_{\mathrm{sc}} / J_{\mathrm{rec}, \mathrm{s}(\mathrm{oc})}$, and from $\eta^{*}=1-R_{\mathrm{rec}, \mathrm{s}(\mathrm{oc})} / R_{\mathrm{rec}, \mathrm{s}(\mathrm{sc})}$ for consistency checking.

\begin{tabular}{|c|c|c|l|}
\hline $\begin{array}{l}\text { Thickness } \\
(\mathbf{n m})\end{array}$ & $\begin{array}{l}\alpha \\
(\%)\end{array}$ & \multicolumn{1}{l|}{$\eta^{*}$} \\
\hline $\begin{array}{l}\text { regular } \\
\mathrm{MAPbI}_{3}\end{array}$ & & 0.993 & 0.990 \\
\hline 471 & 0.7 & 0.984 & 0.980 \\
\hline 364 & 1.6 & 0.988 & 0.988 \\
\hline 300 & 1.2 & & \\
\hline $\begin{array}{l}\text { Regular } \\
\mathrm{FAPbI}_{3-x} \mathrm{Cl}_{x}\end{array}$ & & 0.990 & 0.965 \\
\hline 300 & 1.0 & 0.993 & 0.991 \\
\hline 190 & 0.7 & 0.994 & 0.990 \\
\hline 130 & 1.6 & & \\
\hline
\end{tabular}




\begin{tabular}{|c|c|c|c|}
\hline $\begin{array}{l}\text { Inverted } \\
\mathrm{MAPbI}_{3-x} \mathrm{Cl}_{x}\end{array}$ & & & \\
\hline 400 & 0.8 & 0.992 & - \\
\hline 190 & 1.0 & 0.990 & - \\
\hline
\end{tabular}

Recent experimental reports situate the radiative limit of the open circuit voltage $V_{\text {oc,rad }} \approx 1.31 \mathrm{~V}$ in the case of mixed halide methylammonium-based perovskites. ${ }^{7}$ This implies photovoltage losses $\Delta V_{\mathrm{oc}}=V_{\mathrm{oc}, \text { rad }}-V_{\mathrm{oc}}$ produced by nonradiative carrier recombination at the interfaces in the range of 0.21-0.27 $\mathrm{V}$ for regular cells and of 0.37$0.39 \mathrm{~V}$ in the case of inverted cells (Table 1). The loss in $\Delta V_{\mathrm{oc}} \approx 0.3 \mathrm{~V}$ has to be related to the effect of the recombination current in reducing the carrier density at the contact. But as outlined previously (and sketched in Figure 3) the photovoltage has a significant electrostatic contribution related to the formation of the upward band bending at the accumulation zone. This is mainly governed by the majorities (holes) that should undergo a diminution by recombination. Recalling the surface hole density as $p_{\mathrm{s}}=p_{0} L_{D} \sqrt{2} e^{q V / 2 k_{\mathrm{B}} T}$ on can estimate the relative reduction in surface hole density as $\Delta p_{\mathrm{s}} \approx 4 \times 10^{2}$ with respect to the value reachable in the radiative limit of $V_{\mathrm{oc}}$. Developing novel processing strategies and buffer layers for surface passivation is thus crucial to increase the outer open circuit voltage.

Discussion. The outlined surface recombination model highlights contact properties as determining parameters in the operation of perovskite solar cells. More specifically, we propose here that the most significant part of the nonradiative recombination occurs in the vicinity of the contacts. These claims could be refuted by recalling that impedance techniques cannot discern about specific recombination mechanisms placed either at the bulk or the outer interfaces. However, the dependence on layer thickness exhibited by the resistive components at short circuit reinforces our view. As observed in Figure $2 b$ and $\mathrm{S} 4 \mathrm{~b}$, recombination resistances (per unit area) rather collapse into a unique value. This trend can be hardly understood in terms of a purely bulk (whole perovskite thickness) mechanism, since an internal recombination flux would scale with the recombining material volume and the corresponding resistance should decrease accordingly. A second argument in favor of the interfacial origin for the recombination processes lies on the fact that the recombination resistance behaves in the opposite way than accumulation capacitance so as to render constant response times. The evident coupling between low-frequency impedance response parameters points again to the location of nonradiative recombination effects at the outer interfaces.

The model sketched in Figure 3 locates the recombination events within the accumulation zone formed in the vicinity of the perovskite/contact interface. i.e. recombining electrons and holes concur in the same material. An alternative schema could claim a charge transfer process between electrons in $\mathrm{TiO}_{2}$ and accumulated holes 
in the perovskite, inspired in dye solar cell known mechanisms (Figure 3a). ${ }^{31}$ However, these charge transfer models usually include the effect of energetic disorder on the recombination, which does not seem to be the case here. Indeed, as noted previously, the resistive response at open circuit (Figure 2d) exhibits a variation with $V_{\text {oc }}$ following $-1 / 2 k_{\mathrm{B}} T$ slope, which contains the statistic mark of the majority carriers. This supports our proposed carrier recombination mainly located in the perovskite material, with the contacting layers taking the role of a mere electron reservoir unable to limit the annihilation dynamics. Obviously more experimental data is certainly needed on this issue.

Although our findings are fully consistent with a nonradiative recombination located at the outer interfaces, the proposed model only addresses the contact energetics at open circuit conditions (Figure 3). The built-up of an accumulation zone near the cathode withstands part of the photovoltage, originating prominent capacitive effects such as $C_{\mathrm{s}}$ (Equation 1). However, it is experimentally observed that high capacitances are also encountered in short circuit conditions, as shown in Figure 2a, S4a and 6a. At short circuit the solar cell ought to operate in nearly full depletion conditions. ${ }^{32-33}$ It is then not likely that interface accumulation zone could be similarly formed. The origin of the short circuit, light-induced capacitance remains elusive in many aspects. Some reports have suggested the effect of interface trapping sites on the capacitive and hysteretic response. ${ }^{29}$ Traps can easily accommodate charges and they are believed to be passivated by using fullerene derivatives, explaining the low-frequency capacitance reduction observed in comparing Figure $2 \mathrm{a}$ and $6 \mathrm{a}$. However, the constancy of the response time (Figure 4) suggests us that both short circuit and open circuit impedance responses share common mechanisms. It should be also noted that ionic motion, particularly near the interfaces, is able to produce local changes in the electronic energetic landscape, giving rise to the reported capacitance response. ${ }^{24,34-35}$ To progress on this concern, informative experiments should be devised aiming the coupled interaction between electronic and ionic dynamics.

Conclusion. Based on the analysis of resistances and capacitances of perovskite solar cell under varying conditions of voltage and illumination, we conclude that most significant part of the nonradiative carrier recombination occurs in the vicinity of the contacts. Carrier losses by surface recombination dominate the performance of perovskite solar cells and consequently govern the reduction in both short circuit current and open circuit voltage. The analysis is based on modeling surface kinetic processes through a complete equivalent circuit that gives a full interpretative framework of the impedance response. An essential component of the model is the formation of a hole accumulation zone in the perovskite layer near the contact. A characteristic time of surface recombination is extracted that exhibits a constant value irrespective of the irradiation intensity for a given solar cell structure. The model is checked for several perovskite solar cell architectures, both regular and inverted, comprising different light 
absorber perovskite materials and selective contacts. This approach enables a comparison among different structures, morphologies, processing strategies of passivation and buffer layers. Photocurrent reduction is calculated to be as low as $1 \%$, signaling the good extraction properties of the contacts. Photovoltage loss is connected to the diminution in surface hole density producing the observed $0.3 \mathrm{~V}$-reduction with respect to the radiative limit.

\section{Author Information}

Notes

The authors declare no competing financial interest.

\section{Acknowledgements}

We thank financial support by Ministerio de Economía y Competitividad (MINECO) of Spain under project (MAT2016-76892-C3-1-R), and Generalitat Valenciana (Prometeo/2014/020). SCIC at UJI are also acknowledged.

\section{Associated Content}

Supporting Information Available: Solar cell preparation description, light $J$ - $V$ curves of analyzed structures, and impedance parameters corresponding to $\mathrm{FAPbI}_{3-x} \mathrm{Cl}_{x}$-based solar cells. The Supporting Information is available free of charge on the ACS Publications website at DOI:.

\section{References}

(1) Saliba, M.; Matsui, T.; Seo, J.-Y.; Domanski, K.; Correa-Baena, J.-P.; Nazeeruddin, M. K.; Zakeeruddin, S. M.; Tress, W.; Abate, A.; Hagfeldt, Anders ; Grätzel, M. Cesium-Containing Triple Cation Perovskite Solar Cells: Improved Stability, Reproducibility and High Efficiency. Energy Environ. Sci. 2016, 9, 1989-1997.

(2) Li, X.; Bi, D.; Yi, C.; Décoppet, J.-D.; Luo, J.; Zakeeruddin, S. M.; Hagfeldt, A.; Grätzel, M. A Vacuum Flash-Assisted Solution Process for High-Efficiency Large-Area Perovskite Solar Cells. Science 2016, 353, 58-62.

(3) Stranks, S. D.; Eperon, G. E.; Grancini, G.; Menelaou, C.; Alcocer, M. J. P.; Leijtens, T.; Herz, L. M.; Petrozza, A.; Snaith, H. J. Electron-Hole Diffusion Lengths Exceeding 1 Micrometer in an Organometal Trihalide Perovskite Absorber. Science 2013, 342, 341-344.

(4) Tian, W.; Zhao, C.; Leng, J.; Cui, R.; Jin, S. Visualizing Carrier Diffusion in Individual Single-Crystal Organolead Halide Perovskite Nanowires and Nanoplates. $J$. Am. Chem. Soc. 2015, 137, 12458 - 12461.

(5) Oga, H.; Saeki, A.; Ogomi, Y.; Hayase, S.; Seki, S. Improved Understanding of 
the Electronic and Energetic Landscapes of Perovskite Solar Cells: High Local Charge Carrier Mobility, Reduced Recombination, and Extremely Shallow Traps. J. Am. Chem. Soc. 2014, 136, 13818-13825.

(6) Wehrenfennig, C.; Eperon, G. E.; Johnston, M. B.; Snaith, H. J.; Herz, L. M. High Charge Carrier Mobilities and Lifetimes in Organolead Trihalide Perovskites. Adv. Mater. 2014, 26, 1584-1589.

(7) Hou, Y.; Chen, W.; Baran, D.; Stubhan, T.; Luechinger, N. A.; Hartmeier, B.; Richter, M.; Min, J.; Chen, S.; Ramirez Quiroz, C. O.; Li, N.; Zhang, H.; Heumueller, T.; Matt, G. J.; Osvet, A.; Forberich, K.; Zhang, Z.-G.; Li, Y.; Winter, B.; Schweizer, P.; Spiecker, E.; Brabec, C. J. Overcoming the Interface Losses in Planar Heterojunction Perovskite-Based Solar Cells. Adv. Mater. 2016, 28, 5112-5120.

(8) Lee, Y. H.; Luo, J.; Son, M.-K.; Gao, P.; Cho, K. T.; Seo, J.; Zakeeruddin, S. M.; Grätzel, M.; Nazeeruddin, M. K. Enhanced Charge Collection with Passivation Layers in Perovskite Solar Cells. Adv. Mater. 2016, 28, 3966-3972.

(9) Marin-Beloqui, J. M.; Lanzetta, L.; Palomares, E. Decreasing Charge Losses in Perovskite Solar Cells Through mp-TiO ${ }_{2}$ MAPI Interface Engineering. Chem. Mater. 2016, 28, 207-213.

(10) Fabregat-Santiago, F.; Bisquert, J.; Garcia-Belmonte, G.; Boschloo, G.; Hagfeldt, A. Influence of Electrolyte in Transport and Recombination in Dye-Sensitized Solar Cells Studied by Impedance Spectroscopy. Sol. Energy Mater. Sol. Cells 2005, 87, 117-131.

(11) Garcia-Belmonte, G.; Guerrero, A.; Bisquert, J. Elucidating Operating Modes of Bulk-Heterojunction Solar Cells from Impedance Spectroscopy Analysis. J. Phys. Chem. Lett. 2013, 4, 877-886.

(12) Mora-Seró, I.; Garcia-Belmonte, G.; Boix, P. P.; Vázquez, M. A.; Bisquert, J. Impedance Spectroscopy Characterisation of Highly Efficient Silicon Solar Cells under Different Light Illumination Intensities. Energy Environ. Sci. 2009, 2, 678-686.

(13) Dualeh, A.; Moehl, T.; Tétreault, N.; Teuscher, J.; Gao, P.; Nazeeruddin, M. K.; Grätzel, M. Impedance Spectroscopic Analysis of Lead Iodide Perovskite-Sensitized Solid-State Solar Cells. ACS Nano 2014, 8, 362-373.

(14) Gonzalez-Pedro, V.; Juarez-Perez, E. J.; Arsyad, W.-S.; Barea, E. M.; Fabregat-Santiago, F.; Mora-Sero, I.; Bisquert, J. General Working Principles of $\mathrm{CH}_{3} \mathrm{NH}_{3} \mathrm{PbX}_{3}$ Perovskite Solar Cells. Nano Lett. 2014, 14, 888-893.

(15) Pascoe, A. R.; Duffy, N. W.; Scully, A. D.; Huang, F.; Cheng, Y.-B. Insights into Planar $\mathrm{CH}_{3} \mathrm{NH}_{3} \mathrm{PbI}_{3}$ Perovskite Solar Cells Using Impedance Spectroscopy. J. Phys. Chem. C 2015, 119, 4444-4453.

(16) Pockett, A.; Eperon, G. E.; Peltola, T.; Snaith, H. J.; Walker, A.; Peter, L. M.; 
Cameron, P. J. Characterization of Planar Lead Halide Perovskite Solar Cells by Impedance Spectroscopy, Open-Circuit Photovoltage Decay, and Intensity-Modulated Photovoltage/Photocurrent Spectroscopy. J. Phys. Chem. C 2015, 119, 3456-3465.

(17) Almora, O.; Zarazua, I.; Mas-Marza, E.; Mora-Sero, I.; Bisquert, J.; GarciaBelmonte, G. Capacitive Dark Currents, Hysteresis, and Electrode Polarization in Lead Halide Perovskite Solar Cells. J. Phys. Chem. Lett. 2015, 6, 1645-1652.

(18) Zarazua, I.; Bisquert, J.; Garcia-Belmonte, G. Light-Induced Space-Charge Accumulation Zone as Photovoltaic Mechanism in Perovskite Solar Cells. J. Phys. Chem. Lett. 2016, 7, 525-528.

(19) Juarez-Perez, E. J.; Wußler, M.; Fabregat-Santiago, F.; Lakus-Wollny, K.; Mankel, E.; Mayer, T.; Jaegermann, W.; Mora-Sero, I. Role of the Selective Contacts in the Performance of Lead Halide Perovskite Solar Cells. J. Phys. Chem. Lett. 2014, 5, 680-685.

(20) Guerrero, A.; Garcia-Belmonte, G.; Mora-Sero, I.; Bisquert, J.; Kang, Y. S.; Jacobsson, T. J.; Correa-Baena, J.-P.; Hagfeldt, A. Properties of Contact and Bulk Impedances in Hybrid Lead Halide Perovskite Solar Cells Including Inductive Loop Elements. J. Phys. Chem. C 2016, 120, 8023-8032.

(21) Suarez, B.; Gonzalez-Pedro, V.; Ripolles, T. S.; Sanchez, R. S.; Otero, L.; Mora-Sero, I. Recombination Study of Combined Halides (Cl, Br, I) Perovskite Solar Cells. J. Phys. Chem. Lett. 2014, 5, 1628-1635.

(22) Juarez-Perez, E. J.; Sanchez, R. S.; Badia, L.; Garcia-Belmonte, G.; Kang, Y. S.; Mora-Sero, I.; Bisquert, J. Photoinduced Giant Dielectric Constant in Lead Halide Perovskite Solar Cells. J. Phys. Chem. Lett. 2014, 5, 2390-2394.

(23) Mora-Seró, I.; Luo, Y.; Garcia-Belmonte, G.; Bisquert, J.; Muñoz, D.; Voz, C.; Puigdollers, J.; Alcubilla, R. Recombination Rates in Heterojunction Silicon Solar Cells Analyzed by Impedance Spectroscopy at Forward Bias and under Illumination. Sol. Energy Mater. Sol. Cells 2008, 92, 505-509.

(24) Yang, T.-Y.; Gregori, G.; Pellet, N.; Grätzel, M.; Maier, J. The Significance of Ion Conduction in a Hybrid Organic-Inorganic Lead-Iodide-Based Perovskite Photosensitizer. Angew. Chem. 2015, 127, 8016 -8021.

(25) Zohar, A.; Kedem, N.; Levine, I.; Zohar, D.; Vilan, A.; Ehre, D.; Hodes, G.; Cahen, D. Impedance Spectroscopic Indication for Solid State Electrochemical Reaction in $\left(\mathrm{CH}_{3} \mathrm{NH}_{3}\right) \mathrm{PbI}_{3}$ Films. J. Phys. Chem. Lett. 2016, 7, 191-197.

(26) Würfel, P. Physics of Solar Cells: From Basic Principles to Advanced Concepts. 2nd ed.; Wiley-VCH: Weinheim, 2009.

(27) Bisquert, J. Nanostructured Energy Devices: Equilibrium Concepts and Kinetics. CRC Press Taylor \& Francis Group: Boca Raton, 2014. 
(28) Bisquert, J.; Garcia-Belmonte, G.; Mora-Sero, I. Characterization of Capacitance, Transport and Recombination Parameters in Hybrid Perovskite and Organic Solar Cells. In Unconventional Thin Film Photovoltaics, Como, E. D.; Angelis, F. D.; Snaith, H.; Walker, A., Eds. The Royal Society of Chemistry: London, 2016.

(29) Shao, Y.; Xiao, Z.; Bi, C.; Yuan, Y.; Huang, J. Origin and Elimination of Photocurrent Hysteresis by Fullerene Passivation in $\mathrm{CH}_{3} \mathrm{NH}_{3} \mathrm{PbI}_{3}$ Planar Heterojunction Solar Cells. Nat. Commun. 2014, 5, 5784.

(30) Almora, O.; Aranda, C.; Zarazua, I.; Guerrero, A.; Garcia-Belmonte, G. Noncapacitive Hysteresis in Perovskite Solar Cells at Room Temperature. ACS Energy Lett. 2016, 1, 209-215.

(31) O’Regan, B. C.; Barnes, P. R. F.; Li, X.; Law, C.; Palomares, E.; MarinBeloqui, J. M. Optoelectronic Studies of Methylammonium Lead Iodide Perovskite Solar Cells with Mesoporous $\mathrm{TiO}_{2}$ : Separation of Electronic and Chemical Charge Storage, Understanding Two Recombination Lifetimes, and the Evolution of Band Offsets during J-V Hysteresis. J. Am. Chem. Soc. 2015, 137, 5087-5099.

(32) Guerrero, A.; Juarez-Perez, E. J.; Bisquert, J.; Mora-Sero, I.; Garcia-Belmonte, G. Electrical Field Profile and Doping in Planar Lead Halide Perovskite Solar Cells. Appl. Phys. Lett. 2014, 105, 133902.

(33) Jiang, C.-S.; Yang, M.; Zhou, Y.; To, B.; Nanayakkara, S. U.; Luther, J. M.; Zhou, W.; Berry, J. J.; Lagemaat, J. v. d.; Padture, N. P.; Zhu, K.; Al-Jassim, M. M. Carrier Separation and Transport in Perovskite Solar Cells Studied by Nanometre-Scale Profiling of Electrical Potential. Nat. Commun. 2015, 6, 8397.

(34) Azpiroz, J. M.; Mosconi, E.; Bisquert, J.; De Angelis, F. Defect Migration in Methylammonium Lead Iodide and its Role in Perovskite Solar Cell Operation. Energy Environ. Sci. 2015, 8, 2118-2127.

(35) Almora, O.; Guerrero, A.; Garcia-Belmonte, G. Ionic Charging by Local Imbalance at Interfaces in Hybrid Lead Halide Perovskites. Appl. Phys. Lett. 2016, 108, 043903. 